\section{\$13. Mechanical Behavior of Strands in CIC Conductor Analyzed by Monte Carlo Method}

\section{Nishijima, S. (ISIR. Osaka Univ.)}

\section{i ) Introduction}

For the large scale superconducting magnet, a cablein-conduit (CIC) conductor has been used because it has high cooling capability, high mechanical rigidity, and high insulating capability.1) The quantitative discussion about the frictional heating induced by strand motion and the contact stress between strands of CIC conductor has not been made because the behavior of strands during energizing has not been clarified. If the strand positions in the conduit are clarified, the current imbalance may be also evaluated because the inductance of each strand can be estimated.

To analyze the strand motion in a conduit, the method where the equations of motion of each strand are solved with time has been developed.2) This method, called wire dynamics simulation, was applied to the CIC conductor. However, it takes much time to simulate the strand motion in a large system. The purpose of the work is to develop a new method to simulate the mechanical behavior of strands in larger system. In this work, Monte Carlo method was employed. The behavior of strands during energizing is reported together with the developed method.

\section{ii) Calculation procedure}

The strands were divided into meshes and the interaction between them was calculated. In the analysis, the position of each mesh which gives the minimal potential energy of the system was calculated. Each mesh was moved small distance decided by the random number and then the potential energy was calculated. The potential energy before and after the movement were compared. When the potential energy after the movement was lower than that before, the strand positions were renewed as the more stable positions. When the potential energy after the movement was higher than that before, the strand position was not accepted. The procedure was performed in all meshes. This one run is called ' 1 Monte Carlo Time'. This process was continued until the potential energy come to be stable. Then the stable position as the minimum potential energy can be realized.

The energies considered in this work are 1) 'Est', the strain energy induced by the stretching the strand calculated by Hooke's law, 2) 'Ebend', the bending energy of the strands calculated by the beam theory, 3) 'Econtact', the strain energy between strands due to strand deformation in radial direction, and 4) 'Ereact', the strain energy between strands and conduit. 'Econtact' and 'Ereact' were calculated from Hertz's equation. The electromagnetic energy, 'Eelect' was also considered. The potential energy of each mesh was calculated and the total sum of the potential energy,'Etotal' was defined as the potential energy of the whole cable.

\section{iii) Result and discussion}

The strand motion during energizing the CIC conductor was calculated. The last stage cable was encapsulated in the conduit and the void fraction was set at $38 \%$. In Fig. 1, the stable position of strands encapsulated in the conduit is presented. Next the CIC conductor energized up to $31.3 \mathrm{kA}$ in the external field of $5 \mathrm{~T}$. An external magnetic field is vertically applied in $+Y$ direction considering the coil radius is much larger than the conduit size. Lorentz force applies to strands in $+X$ direction. The position of strands at $31.3 \mathrm{kA}$ of transport current is shown in Fig. 2. Each strand moves in $+X$ direction and the strands are pressed to the conduit wall and some of strands are in contact with the conduit wall. The density of the strands near right edge is higher than that near left edge in the conduit. These results can explain the phenomenon occurred in the CIC conductor. Using this method the stability of the CIC conductor would be evaluated.

\section{iv) Conclusion}

A method to evaluate the strand position in the CIC conductor has been developed. The method developed in this work enables to calculate the stable position of the strands, the movement of strands during energizing, the distribution of contact stress between strands, and the loaddisplacement curves of the cable. It is expected that the method has the possibility to give the design standard for the stable CIC conductor.

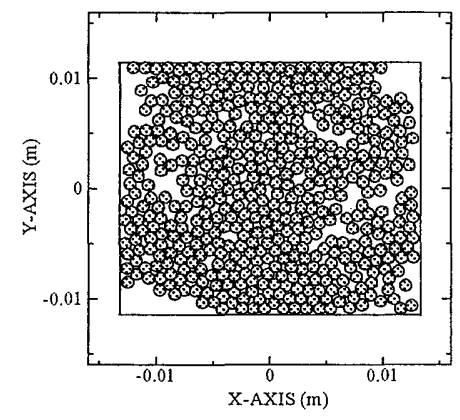

Fig. 1. Position of strands before energizing (void fraction $38 \%)$

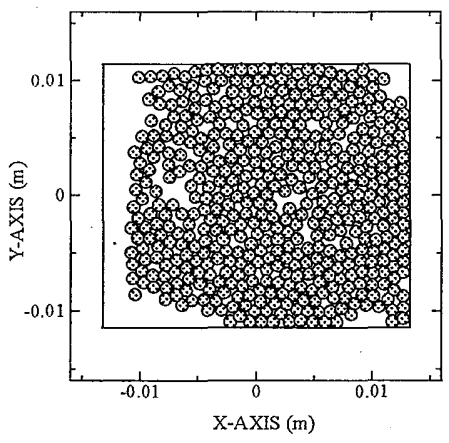

Fig.2. Position of strands during energizing (transport current $31.3 \mathrm{kA}$ and external field 5T)

Reference

1) J.W.Lue, J.Appl.Phys 51, (1980) 772

2) Nishijima, S., IEEE Transactions on Applied Superconductivity 7, (1997) 163 